\title{
CONVOLUTION OF ALMOST CHARACTERS*
}

\author{
G. LUSZTIG ${ }^{\dagger}$
}

0. In this paper we show that the convolution product of "almost characters" of a connected reductive group over a finite field is given by "structure constants" whose leading coefficients can be interpreted in $K$-theoretic terms and in particular are natural numbers.

I wish to thank M. Finkelberg and V. Ostrik for comments on an earlier version of this paper.

1. Let $\mathcal{G}$ be a finite group. Let $\operatorname{Vec}_{\mathcal{G}}$ be the category whose objects are $\mathbf{C}$-vector bundles on $\mathcal{G}$ which are $\mathcal{G}$-equivariant for the conjugation action of $\mathcal{G}$ on $\mathcal{G}$. For $U \in \operatorname{Vec}_{\mathcal{G}}$ let $U_{g}$ denote the fibre of $U$ at $g \in \mathcal{G}$; the $\mathcal{G}$-equivariant structure on $U$ is given by isomorphisms $\psi_{x}^{U}: U_{y} \rightarrow U_{x y x^{-1}}$ for any $x, y \in \mathcal{G}$. For $U, U^{\prime} \in \operatorname{Vec}_{\mathcal{G}}$ we define $U * U^{\prime} \in \operatorname{Vec}_{\mathcal{G}}$ as follows: for $y \in \mathcal{G}$, we have $\left(U * U^{\prime}\right)_{y}=\oplus_{\left(g, g^{\prime}\right) \in \mathcal{G} \times \mathcal{G} ; g g^{\prime}=y} U_{g} \otimes U_{g^{\prime}}^{\prime}$; the $\mathcal{G}$-equivariant structure on $U * U^{\prime}$ is defined by

$$
\psi_{x}^{U * U^{\prime}}: \oplus_{\left(g, g^{\prime}\right) \in \mathcal{G} \times \mathcal{G} ; g g^{\prime}=y} U_{g} \otimes U_{g^{\prime}}^{\prime} \rightarrow \oplus_{\left(h, h^{\prime}\right) \in \mathcal{G} \times \mathcal{G} ; h h^{\prime}=x y x^{-1}} U_{h} \otimes U_{h^{\prime}}^{\prime}
$$

given by the direct sum over $\left\{\left(g, g^{\prime}\right) \in \mathcal{G} \times \mathcal{G} ; g g^{\prime}=y\right\}$ of $\psi_{x}^{U} \otimes \psi_{x}^{U^{\prime}}: U_{g} \otimes U_{g^{\prime}}^{\prime} \rightarrow$ $U_{x g x^{-1}} \otimes U_{x g^{\prime} x^{-1}}^{\prime}$. Then $\operatorname{Vec}_{\mathcal{G}}$ becomes a tensor category. In [L4, p.242] it is shown that $U * U^{\prime}$ is isomorphic to $U^{\prime} * U$ for any $U, U^{\prime} \in \operatorname{Vec}_{\mathcal{G}}$. More directly, for any $y \in \mathcal{G}$ we define an isomorphism

$$
\pi_{y}: \oplus_{\left(g, g^{\prime}\right) \in \mathcal{G} \times \mathcal{G} ; g g^{\prime}=y} U_{g} \otimes U_{g^{\prime}}^{\prime} \rightarrow \oplus_{\left(h, h^{\prime}\right) \in \mathcal{G} \times \mathcal{G} ; h h^{\prime}=y} U_{h}^{\prime} \otimes U_{h^{\prime}}
$$

by $\pi_{y}\left(u \otimes u^{\prime}\right)=\psi_{g}^{U^{\prime}}\left(u^{\prime}\right) \otimes u \in U_{g g^{\prime} g^{-1}}^{\prime} \otimes U_{g}$ (where $\left.u \in U_{g}, u^{\prime} \in U_{g^{\prime}}^{\prime}\right)$. Then $\pi=$ $\left(\pi_{y}\right)_{y \in \mathcal{G}}: U * U^{\prime} \rightarrow U^{\prime} * U$ is an isomorphism of $\mathcal{G}$-equivariant vector bundles.

Let $\mathcal{M}(\mathcal{G})$ be the set of pairs $(g, \rho)$ where $g \in \mathcal{G}$ is defined up to $\mathcal{G}$-conjugacy and $\rho$ is an irreducible representation of the centralizer $Z_{\mathcal{G}}(g)$ defined up to isomorphism. As in [L1] we have a pairing $\{\}:, \mathcal{M}(\mathcal{G}) \times \mathcal{M}(\mathcal{G}) \rightarrow \mathbf{C}$ given by

$$
\left\{(g, \rho),\left(g^{\prime}, \rho^{\prime}\right)\right\}=\left|Z_{\mathcal{G}}(g)\right|^{-1}\left|Z_{\mathcal{G}}\left(g^{\prime}\right)\right|^{-1} \sum_{h \in \mathcal{G} ; h^{-1} g h \in Z_{\mathcal{G}}\left(g^{\prime}\right)} \operatorname{tr}\left(h g^{\prime} h^{-1}, \rho\right) \operatorname{tr}\left(h^{-1} g^{-1} h, \rho^{\prime}\right) .
$$

An object in $\mathrm{Vec}_{\mathcal{G}}$ is said to be irreducible if it is non-zero and is not a direct sum of two non-zero objects of $\mathrm{Vec}_{\mathcal{G}}$. According to [L4,2.5], the set of isomorphism classes of irreducible objects of $\operatorname{Vec}_{\mathcal{G}}$ is in natural bijection with the set $\mathcal{M}(\mathcal{G})$ : to $m=$ $(g, \rho) \in \mathcal{M}(\mathcal{G})$ corresponds an object $U=U^{m} \in \operatorname{Vec}_{\mathcal{G}}$ such that $U_{h}$ is zero for $h \in \mathcal{G}$ not conjugate to $g$ and $\operatorname{tr}\left(\psi_{x}^{U}: U_{g} \rightarrow U_{g}\right)=\operatorname{tr}(x, \rho)$ for any $x \in Z_{\mathcal{G}}(g)$. Then $\left(U^{m}\right)_{m \in \mathcal{M}(\mathcal{G})}$ is a $\mathbf{Z}$-basis for the Grothendieck group $K_{\mathcal{G}}(\mathcal{G})$ of $\operatorname{Vec}_{\mathcal{G}}$. For $m, m^{\prime}$ in $\mathcal{M}(\mathcal{G})$, we can write

$$
U^{m} * U^{m^{\prime}} \cong \oplus_{m^{\prime \prime} \in \mathcal{M}(\mathcal{G})}\left(U^{m^{\prime \prime}}\right)^{\oplus p_{m, m^{\prime}, m^{\prime \prime}}}
$$

* Received January 21, 2004; accepted for publication June 4, 2004.

${ }^{\dagger}$ Department of Mathematics, M.I.T., Cambridge, MA 02139, USA (gyuri@math.mit.edu). Supported in part by the National Science Foundation. 
where $p_{m, m^{\prime}, m^{\prime \prime}} \in \mathbf{N}$ are multiplicities. Now $*$ induces a structure of commutative ring on $K_{\mathcal{G}}(\mathcal{G})$ in which $p_{m, m^{\prime}, m^{\prime \prime}}$ are structure constants:

$$
U_{m} U_{m^{\prime}}=\sum_{m^{\prime \prime} \in \mathcal{M}(\mathcal{G})} p_{m, m^{\prime}, m^{\prime \prime}} U_{m^{\prime \prime}}
$$

According to $[\mathrm{L} 4,2.5(\mathrm{~d})]$ for any $(z, \eta) \in \mathcal{M}(\mathcal{G})$, the assignment

$$
U^{m} \mapsto(\operatorname{dim} \eta)^{-1}\left|Z_{\mathcal{G}}(z)\right|\{m,(z, \eta)\}
$$

defines a ring homomorphism $K_{\mathcal{G}}(\mathcal{G}) \rightarrow \mathbf{C}$. Applying this ring homomorphism to (a) gives

$$
(\operatorname{dim} \eta)^{-1}\left|Z_{\mathcal{G}}(z)\right|\{m,(z, \eta)\}\left\{m^{\prime},(z, \eta)\right\}=\sum_{m^{\prime \prime} \in \mathcal{M}(\mathcal{G})} p_{m, m^{\prime}, m^{\prime \prime}}\left\{m^{\prime \prime},(z, \eta)\right\} .
$$

Since the matrix $\left(\left\{m, m^{\prime}\right\}\right)$ has square 1 (see [L1]) we deduce that

$$
\text { (b) } \quad p_{m, m, m^{\prime \prime}}=\sum_{(z, \eta) \in \mathcal{M}(\mathcal{G})}(\operatorname{dim} \eta)^{-1}\left|Z_{\mathcal{G}}(z)\right|\{m,(z, \eta)\}\left\{m^{\prime},(z, \eta)\right\}\left\{(z, \eta), m^{\prime \prime}\right\} .
$$

2. Let $\Gamma$ be a finite group and let $f, f^{\prime}$ be two class functions $\Gamma \rightarrow \mathbf{C}$. Recall that the convolution $f * f^{\prime}$ is the class function $\Gamma \rightarrow \mathbf{C}$ given by $\left(f * f^{\prime}\right)(\gamma)=$ $\sum_{\gamma_{1} \in \Gamma} f\left(\gamma_{1}\right) f^{\prime}\left(\gamma_{1}^{-1} \gamma\right)$. It is well known that given irreducible characters $f, f^{\prime}$ of $\Gamma$, we have $f * f^{\prime}=0$ if $f \neq f^{\prime}$ and $f * f=(|\Gamma| / f(1)) f$.

3. Let $G$ be a connected reductive algebraic group over an algebraic closure of a finite field $\mathbf{F}_{q}$, with a fixed $\mathbf{F}_{q}$-rational structure. The vector space of class functions $G\left(\mathbf{F}_{q}\right) \rightarrow \mathbf{C}$ has an orthonormal basis consisting of the irreducible characters and also an orthonormal basis consisting of "almost characters" (see [L2, 13.6]), closely related to character sheaves. We would like to study the convolution of two almost characters. Since the basis of almost characters differs from the basis of irreducible characters by an explicitly known almost diagonal matrix, we see that the convolution of two almost characters is a linear combination of a small number of almost characters. We want to make explicit the coefficients in this linear combination. For simplicity, here we restrict ourselves to "unipotent almost characters"; these are the almost characters that are linear combinations of unipotent characters (they form a basis of the space spanned by the unipotent characters). We also assume that $G$ is split over $\mathbf{F}_{q}$ and that $G$ modulo its centre is simple.

To each two sided cell $\underline{c}$ of the Weyl group $W$ of $G$ we attach as in [L2, §4] a certain finite group $\mathcal{G}_{\underline{c}}$. As in [L2] the set of unipotent characters (resp. unipotent almost characters) of $G^{F}$ can be indexed as $\left(\sigma_{\underline{c}, m}\right)$ (resp. $\left.\left(\hat{\sigma}_{\underline{c}, m}\right)\right)$ where $\underline{c}$ is a two sided cell of $W, m \in \mathcal{M}\left(\mathcal{G}_{\underline{c}}\right)$ and

$$
\begin{aligned}
\hat{\sigma}_{\underline{c}, m} & =\sum_{m^{\prime} \in \mathcal{M}\left(\mathcal{G}_{\underline{c}}\right)}\left\{m^{\prime}, m\right\} \Delta\left(\underline{c}, m^{\prime}\right) \sigma_{\underline{c}, m^{\prime}} \\
\sigma_{\underline{c}, m} & =\sum_{m^{\prime} \in \mathcal{M}\left(\mathcal{G}_{\underline{c}}\right)}\left\{m^{\prime}, m\right\} \Delta(\underline{c}, m) \hat{\sigma}_{\underline{c}, m^{\prime}}
\end{aligned}
$$

with $\Delta\left(\underline{c}, m^{\prime}\right)= \pm 1$. Moreover, to any two-sided cell $\underline{c}$ one can attach an integer $A_{\underline{c}} \geq 0$ such that for any $m=(g, \rho) \in \mathcal{G}_{\underline{c}},\left|G\left(\mathbf{F}_{q}\right)\right| / \sigma_{\underline{c}, m}(1)$ is a polynomial in $q$ with 
constant rational coefficients of the form

$$
\frac{\left|Z_{\mathcal{G}_{\underline{c}}}(g)\right|}{\operatorname{dim} \rho} q^{D-A_{\underline{c}}}+\text { lower powers of } q .
$$

$($ Here $D=\operatorname{dim} G$.)

We now consider two unipotent almost characters $\hat{\sigma}_{\underline{c}, m}, \hat{\sigma}_{\underline{c}^{\prime}, m^{\prime}}$. From (a) we see that

$$
\hat{\sigma}_{\underline{c}, m} * \hat{\sigma}_{\underline{c}^{\prime}, m^{\prime}}=0 \text { if } \underline{c} \neq \underline{c}^{\prime} .
$$

Now assume that $\underline{c}=\underline{c}^{\prime}$. From (a),(b) we have

$$
\begin{aligned}
\hat{\sigma}_{\underline{c}, m} * \hat{\sigma}_{\underline{c}, m^{\prime}} & \sum_{m_{1}, m_{2} \in \mathcal{M}\left(\mathcal{G}_{\underline{c}}\right)}\left\{m_{1}, m\right\}\left\{m_{2}, m^{\prime}\right\} \Delta\left(\underline{c}, m_{1}\right) \Delta\left(\underline{c}, m_{2}\right) \sigma_{\underline{c}, m_{1}} * \sigma_{\underline{c}, m_{2}} \\
= & \sum_{m_{1} \in \mathcal{M}\left(\mathcal{G}_{\underline{c}}\right)}\left\{m_{1}, m\right\}\left\{m_{1}, m^{\prime}\right\}\left|G\left(F_{\mathbf{q}}\right)\right| \sigma_{\underline{c}, m_{1}}(1)^{-1} \sigma_{\underline{c}, m_{1}} \\
= & \sum_{m_{1} \in \mathcal{M}\left(\mathcal{G}_{\underline{c}}\right)}\left\{m_{1}, m\right\}\left\{m_{1}, m^{\prime}\right\}\left|G\left(F_{\mathbf{q}}\right)\right| \sigma_{\underline{c}, m_{1}}(1)^{-1} \\
& \sum_{m^{\prime \prime} \in \mathcal{M}\left(\mathcal{G}_{\underline{c}}\right)}\left\{m^{\prime \prime}, m_{1}\right\} \Delta\left(\underline{c}, m_{1}\right) \hat{\sigma}_{\underline{c}, m^{\prime \prime}} .
\end{aligned}
$$

Thus

$$
\hat{\sigma}_{\underline{c}, m} * \hat{\sigma}_{\underline{c}, m^{\prime}}=\sum_{m_{1}^{\prime} \in \mathcal{M}\left(\mathcal{G}_{\underline{G}}\right)} N_{m, m^{\prime}, m^{\prime \prime}} \hat{\sigma}_{\underline{c}, m^{\prime \prime}}
$$

where, for $m, m^{\prime}, m^{\prime \prime} \in \mathcal{M}\left(\mathcal{G}_{\underline{c}}\right)$ we have

$$
N_{m, m^{\prime}, m^{\prime \prime}}=\sum_{m_{1} \in \mathcal{M}\left(\mathcal{G}_{\underline{c}}\right)}\left\{m_{1}, m\right\}\left\{m_{1}, m^{\prime}\right\}\left\{m^{\prime \prime}, m_{1}\right\} \Delta\left(\underline{c}, m_{1}\right)\left|G\left(F_{\mathbf{q}}\right)\right| \sigma_{\underline{c}, m_{1}}(1)^{-1} .
$$

Using (c) we see that $N_{m, m^{\prime}, m^{\prime \prime}}$ is a polynomial in $q$ with constant coefficients of the form

$$
n_{m, m^{\prime}, m^{\prime \prime}} q^{D-A_{\underline{c}}}+\text { lower powers of } q
$$

where

$$
n_{m, m^{\prime}, m^{\prime \prime}}=\sum_{(z, \eta) \in \mathcal{M}\left(\mathcal{G}_{\underline{c}}\right)} \frac{\left|Z_{\mathcal{G}_{\underline{c}}}(z)\right|}{\operatorname{dim} \eta} \Delta(\underline{c},(z, \eta))\{(z, \eta), m\}\left\{(z, \eta), m^{\prime}\right\}\left\{m^{\prime \prime},(z, \eta)\right\} .
$$

If $\Delta\left(\underline{c}, m_{1}\right)$ is 1 for any $m_{1} \in \mathcal{M}\left(\mathcal{G}_{\underline{c}}\right)$ then, using $1(\mathrm{~b})$ and the identity $\left\{m_{1}, m_{2}\right\}=$ $\overline{\left\{m_{2}, m_{1}\right\}}$, we see that

$$
n_{m, m^{\prime}, m^{\prime \prime}}=p_{m, m^{\prime}, m^{\prime \prime}}
$$

where $p_{m, m^{\prime}, m^{\prime \prime}}$ is defined as in Section 1 in terms of $\mathcal{G}=\mathcal{G}_{\underline{\underline{c}}}$. (We use that the integer $p_{m, m^{\prime}, m^{\prime \prime}}$ is fixed by complex conjugation.) Thus, the leading coefficient $n_{m, m^{\prime}, m^{\prime \prime}}$ has a $K$-theoretic interpretation; in particular, it is in $\mathbf{N}$.

A similar result holds when $m_{1} \mapsto \Delta\left(\underline{c}, m_{1}\right)$ is not identically 1 . (Then $G$ is of type $E_{7}$ and $\underline{c}$ is a two-sided cell with $|\underline{c}|=2 \times 512^{2}$ or $G$ is of type $E_{8}$ and $\underline{c}$ is a two-sided cell with $|\underline{c}|=2 \times 4096^{2}$.) In this case $\mathcal{G}_{\underline{c}}=\mathbf{Z} / 2 \mathbf{Z}, \mathcal{M}_{\mathcal{G}_{\underline{c}}}$ may be identified with the (additive) abelian group $\mathbf{Z} / 2 \mathbf{Z} \times \operatorname{Hom}\left(\mathbf{Z} / 2 \mathbf{Z}, \mathbf{C}^{*}\right)$ and $\Delta\left(\underline{c}, m_{1}\right)=2\left\{m_{0}, m_{1}\right\}$ 
where $m_{0}=(0, \epsilon)$ and $\epsilon \neq 0$. Moreover, in this case we have $\left\{m_{1}, m_{2}\right\}\left\{m_{1}, m_{3}\right\}=$ $\left\{m_{1}, m_{2}+m_{3}\right\} / 2$ and $\left\{m_{1}, m_{2}\right\}=\left\{m_{2}, m_{1}\right\}$. It follows that

$$
n_{m, m^{\prime}, m^{\prime \prime}}=\sum_{m_{1} \in \mathcal{M}\left(\mathcal{G}_{\underline{c}}\right)}\left\{m_{1}, m+m^{\prime}+m^{\prime \prime}+m_{0}\right\} / 2 .
$$

On the other hand we have

$$
p_{m, m^{\prime}, m^{\prime \prime}}=\sum_{m_{1} \in \mathcal{M}\left(\mathcal{G}_{\underline{c}}\right)}\left\{m_{1}, m+m^{\prime}+m^{\prime \prime}\right\} / 2 .
$$

Hence

$$
n_{m, m^{\prime}, m^{\prime \prime}}=p_{m+m_{0}, m^{\prime}+m_{0}, m^{\prime \prime}+m_{0}} .
$$

Remark. If $G$ is a classical group, then for any $\underline{c}, \mathcal{M}\left(\mathcal{G}_{\underline{c}}\right)$ may be regarded as an $\mathbf{F}_{2}$-vector space of even dimension and for $m, m^{\prime}, m^{\prime \prime}$ in $\mathcal{M}\left(\mathcal{G}_{\underline{c}}\right)$ we have $n_{m, m^{\prime}, m^{\prime \prime}}=$ $p_{m, m^{\prime}, m^{\prime \prime}}=1$ if $m+m^{\prime}+m^{\prime \prime}=0$ and $n_{m, m^{\prime}, m^{\prime \prime}}=p_{m, m^{\prime}, m^{\prime \prime}}=0$ if $m+m^{\prime}+m^{\prime \prime} \neq 0$.

4. Let $\underline{c}$ be a two-sided cell of $W$. Let $\mathcal{C}_{\underline{c}}$ be the category with objects of the form $A=A^{\prime}[-D]$ where $A^{\prime}$ is a perverse sheaf on $G$ which is a direct sum of "unipotent" character sheaves corresponding to $\underline{c}$, see [L3]. For $A, B \in \mathcal{C}_{\underline{c}}$ the convolution $A * B$ is by definition $\mu_{!}(A \otimes B)$ where $\mu: G \times G \rightarrow G$ is multiplication. Let $(A * B)^{i}$ be the $i$-th perverse cohomology sheaf of $A * B$. We may regard $A, B$ as mixed complexes, pure of weight 0 . Then $(A * B)^{i}$ has a weight filtration whose associated graded is canonically of the form

$$
\oplus_{j \in \mathbf{Z}}(A * B)^{i, j}(-j / 2)=\oplus_{j^{\prime} \in \mathbf{Z}}(A * B)_{j^{\prime}}^{i}\left(-j^{\prime} / 2\right)
$$

where $(A * B)^{i, j}$ is perverse, pure of weight $0,(-j / 2)$ is a Tate twist and

$$
(A * B)_{j^{\prime}}^{i}=(A * B)^{i, j^{\prime}-D}[-D](-D / 2) .
$$

Moreover, $(A * B)_{j^{\prime}}^{i}$ does not depend on the choice of mixed structures. We expect that $(A * B)_{j^{\prime}}^{i}=0$ if $j^{\prime}>2 D-2 A_{\underline{c}}$ and that $(A * B)_{2 D-2 A_{\underline{c}}}^{i}$ is zero except for at most one value of $i$. We set $A \circ B=\oplus_{i}(A * B)_{2 D-2 A_{\underline{c}}}^{i}$. We expect that $A \circ B \in \mathcal{C}_{\underline{c}}$ and that $A, B \mapsto A \circ B$ defines on $\mathcal{C}_{\underline{c}}$ a structure of tensor category. The results in Section 3 suggest that this tensor category should be equivalent to $\operatorname{Vec}_{\mathcal{G}_{\underline{c}}}$. One evidence for this is the geometric interpretation of the indexing of character sheaves in $\mathcal{C}_{\underline{c}}$ by irreducible vector bundles in $\mathrm{Vec}_{\mathcal{G}_{\underline{c}}}$ indicated in [L3, 4.7(a)].

\section{REFERENCES}

[L1] G.LuszTig, Unipotent representations of a finite Chevalley group of type E8, Quart.J.Math., 30 (1979), pp. 315-338.

[L2] G.LuszTig, Characters of reductive groups over a finite field, Ann. Math. Studies 107, Princeton Univ.Press, 1984.

[L3] G.LuszTig, Introduction to character sheaves, Proc.Symp.Pure Math., 47(1) (1987), pp. 165180.

[L4] G.Lusztig, Leading coefficients of character values of Hecke algebras, Proc.Symp.Pure Math., 47(2) (1987), pp. 235-262. 